\title{
ONE MODULO N GRACEFULNESS OF REGULAR BAMBOO TREE AND COCONUT TREE
}

\author{
V.Ramachandran1 C.Sekar2 \\ 1 Department of Mathematics, P.S.R Engineering College (Affiliated to Anna University \\ Chennai), Sevalpatti, \\ Sivakasi, Tamil Nadu, India. \\ 2 Department of Mathematics, Aditanar College of Arts and Science (Affiliated to MS \\ University Tirunelveli), \\ Tiruchendur, Tamil Nadu, India.
}

\begin{abstract}
A function $\mathrm{f}$ is called a graceful labelling of a graph $\mathrm{G}$ with q edges if $\mathrm{f}$ is an injection from the vertices of $G$ to the set $\{0,1,2, \ldots, q\}$ such that, when each edge $x y$ is assigned the label $|f(x)-f(y)|$, the resulting edge labels are distinct. A graph $G$ is said to be one modulo $\mathrm{N}$ graceful (where $\mathrm{N}$ is a positive integer) if there is a function $\varphi$ from the vertex set of $\mathrm{G}$ to $\{0,1, \mathrm{~N},(\mathrm{~N}+1), 2 \mathrm{~N},(2 \mathrm{~N}+1), \ldots, \mathrm{N}(\mathrm{q}-1), \mathrm{N}(\mathrm{q}-1)+1\}$ in such a way that (i) $\varphi$ is $1-1$ (ii) $\varphi$ induces a bijection $\varphi_{-}$from the edge set of $\mathrm{G}$ to $\{1, \mathrm{~N}+1,2 \mathrm{~N}+1, \ldots, \mathrm{N}(\mathrm{q}-1)+1\}$ where $\varphi_{-}(\mathrm{uv})=|\varphi(\mathrm{u})-\varphi(\mathrm{v})|$. In this paper we prove that the every regular bamboo tree and coconut tree are one modulo $\mathrm{N}$ graceful for all positive integers $\mathrm{N}$.
\end{abstract}

\section{INTRODUCTION}

S.W.Golomb [2] introduced graceful labelling. Odd gracefulness was introduced by. B.Gnanajothi [1] . C.Sekar [6] introduced one modulo three graceful labelling. V.Ramachandran and C.Sekar [4] introduced the concept of one modulo $\mathrm{N}$ graceful where $\mathrm{N}$ is any positive integer. In the case $\mathrm{N}=2$, the labelling is odd graceful and in the case $\mathrm{N}=1$ the labelling is graceful. [6] Every regular bamboo tree is graceful. In this paper we establish the result for one modulo $\mathrm{N}$ graceful $(\mathrm{N}>1)$ of the regular bamboo tree and also we prove that coconut tree is one modulo $\mathrm{N}$ graceful for all positive integers $\mathrm{N}$. In order to prove the existing conjecture

Problem 1. All trees are graceful?

Problem 2. All lobsters are graceful?

we take a diversion to prove one modulo $\mathrm{N}$ graceful of acyclic graphs. Sometimes the technique involved in one modulo $\mathrm{N}$ graceful labelling may yield a new approach to have graceful labelling of graphs. Our approach will motivate the scholars to do more research in this area. 
International journal on applications of graph theory in wireless ad hoc networks and sensor networks (GRAPH-HOC) Vol.6, No.2, June 2014

2 Main Results Definition 2.1. A graph $\mathrm{G}$ with q edges is said to be one modulo $\mathrm{N}$ graceful (where $\mathrm{N}$ is a positive integer) if there is a function $\varphi$ from the vertex set of $\mathrm{G}$ to $\{0,1, \mathrm{~N},(\mathrm{~N}+1)$, $2 \mathrm{~N},(2 \mathrm{~N}+1), \ldots, \mathrm{N}(\mathrm{q}-1), \mathrm{N}(\mathrm{q}-1)+1\}$ in such a way that (i) $\varphi$ is $1-1$ (ii) $\varphi$ induces a bijection $\varphi_{-}$from the edge set of $\mathrm{G}$ to $\{1, \mathrm{~N}+1,2 \mathrm{~N}+1, \ldots, \mathrm{N}(\mathrm{q}-1)+1\}$ where $\varphi_{-}(\mathrm{uv})=\mid \varphi(\mathrm{u})-$ $\varphi(v) \mid$.

Definition 2.2. Consider k copies of paths Pn of length $n-1$ and stars Sm with m pendant vertices. Identify one of the two pendant vertices of the $j$ th path with the centre of the $j$ th star. Identify the other pendant vertex of each path with a single vertex $\mathrm{u} 0 \mathrm{(} \mathrm{u} 0$ is not in any of the star and path).The graph obtained is a regular bamboo tree. Definition 2.3. A coconut Tree $\mathrm{CT}(\mathrm{m}, \mathrm{n})$ is the graph obtained from the path Pn by appending $\mathrm{m}$ new pendent edges at an end vertex of Pn . Theorem 2.4. Every regular bamboo tree is one modulo $\mathrm{N}$ graceful for every positive integer $\mathrm{N}>$ 1 .

Proof: Let $u_{1}^{(j)}, u_{2}^{(j)} \ldots, u_{n}^{(j)}$ be the vertices of the $j$ th path where $u_{1}^{(j)}$ is identificd with $u_{0}$ an $u_{n}^{(j)}$ is identified with $v_{0}^{(j)}$ which is the centre of the $j$ th star. Let $v_{1}^{(j)}, v_{2}^{(j)} \ldots, u_{n}^{(j)}$ be the pendar. vertices of the $j$ th star. The bamboo tree has $k(n+m-1)+1$ vertices and $k(n+m-1)$ edges. Namin of the vertices is as shown in the figure.

Case (i) $\mathrm{k}$ is odd and $\mathrm{n}$ is odd

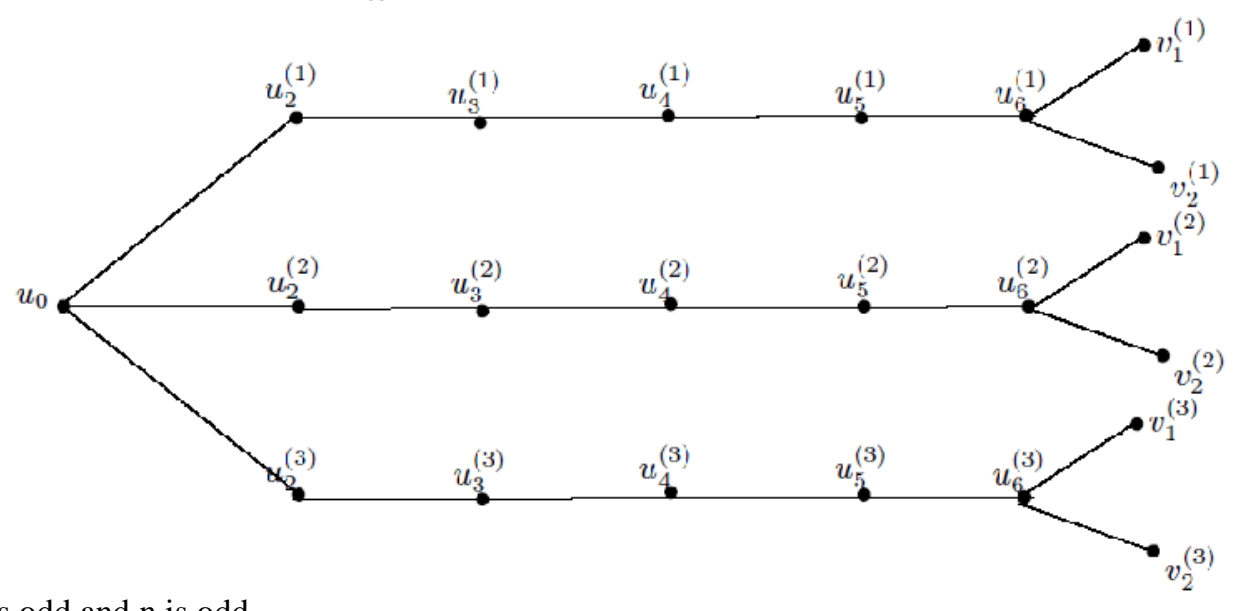

Define 
International journal on applications of graph theory in wireless ad hoc networks and sensor networks

$\phi\left(u_{0}\right)=\phi\left(u_{1}^{\prime \prime \prime}\right)=0$

(GRAPH-HOC) Vol.6, No.2, June 2014

For $i=2,4, \ldots, n-1$

$\phi\left(u_{i}^{(j)}\right)=N k(n+m-1)-(N-1)-N(j-1)-\frac{N k(i-2)}{2}$ for $j=1,2, \ldots, k$

For $i=3,5, \ldots, n$

$\phi\left(u_{i}^{(j)}\right)- \begin{cases}N(k+1)+N(j-1)+\frac{N k(i-3)}{2} & \text { for } j-1,2, \ldots, \frac{(k-1)}{2} \\ \frac{N(k+1)}{2}+N\left(j-\frac{(k+1)}{2}\right)+\frac{N_{k}(i-3)}{2} & \text { for } j=\frac{(k+1)}{2}, \frac{(k+3)}{2}, \ldots, k\end{cases}$

For $r-1,2, \ldots, m$

$\phi\left(v_{r}^{(j)}\right)-N k(n+m-1)-(N-1)-\frac{N k(n-1)}{2}-N k(r-1)-N(j-1)$ for $j-1,2, \ldots, k$

From the definition of $\phi$ it is clear that

$\left\{\phi\left(u_{0}\right)\right\} \cup\left\{\phi\left(u_{i}^{(j)}\right), \quad i=2,3, \ldots, n\right.$ and $\left.j=1,2, \ldots, k\right\} \cup\left\{\phi\left(v_{r}^{(j)}\right), r=1,2, \ldots, m\right.$ and $j=$ $1,2, \ldots, k\}=\{\cup\} \cup\left\{N k(n+m-1)-N+1, N k(n+m-2)-N+1, \ldots, N k\left(\frac{n+2 m+1}{2}\right)-N+\right.$ $1, N k(n+m-1)-2 N+1, N k(n+m-2)-2 N+1, \ldots, N k\left(\frac{n+2 m+1}{2}\right)-2 N+1, \ldots, N k(n+m-$ $\left.2)+1, N k(n+m-3)+1, \ldots, N k\left(\frac{n+2 m-1}{2}\right)+1\right\}()\left\{\mathrm{N}(\mathrm{k}+1), \mathrm{N}(2 \mathrm{k}+1), \ldots, \mathrm{Nk}\left(\frac{n-1}{2}\right)+N, N(k+\right.$ 2), $N(2 k+2), \ldots, N k\left(\frac{n 1}{2}\right)+2 N, \ldots, \frac{N}{2}(3 k-1), \frac{N}{2}(5 k-1), \ldots, \frac{N}{2}(n k-1), \frac{N}{2}(k+1), \quad \frac{N}{2}(3 k+1)$, $\left.\ldots, \quad \frac{N}{2}(n k-k+1), \frac{N}{2}(k+3), \quad \frac{N}{2}(3 k-3),, \ldots, \frac{N}{2}(n k-2 k+3), \ldots, N k, 2 N k, \ldots, \frac{N k}{2}(n-1)\right\} \cup\{$ $\frac{N k}{2}(n+2 m-1)-N+1, \frac{N k}{2}(n+2 m-3)-N+1, \ldots, \frac{N k}{2}(n+1)-N+1, \frac{N k}{2}(n+2 m-1)-2 N+1, \frac{N k}{2}(n+$ $\left.2 m-3)-2 N+1, \ldots, \frac{N k}{2}(n+1)-2 N+1, \ldots, \frac{N k}{2}(n+2 m-3)+1, \frac{N k}{2}(n+2 m-5)+1, \ldots, \frac{N k}{2}(n-1)+1\right\}$

Thus it is clear that the vertices have distinct labels. Therefore $\phi$ is $1-1$.

We compute the edge labelling in the following sequence.

For $1 \leq j \leq k$

$\left|\phi\left(u_{2}^{(j)}\right)-\phi\left(u_{0}\right)\right|=N k(n+m-1)-N j+1$

For $1 \leq r \leq m$ and $j=1,2, \ldots, \frac{k-1}{2}$

$\left|\phi\left(v_{r}^{(j)}\right)-\phi\left(u_{n}^{(j)}\right)\right|=N k(m-r+1)-2 N j+1$

For $1 \leq r \leq m$ and $j=\frac{k+1}{2}, \frac{k+3}{2}, \ldots, k$

$\left|\phi\left(v_{r}^{(j)}\right)-\phi\left(u_{n}^{(j)}\right)\right|=N k(m-r+2)-2 N j+1$

For $j=2,4, \ldots, n-1$ and $j=1,2, \ldots \frac{k-1}{2}$

$\left|\phi\left(u_{i}^{(j)}\right)-\phi\left(u_{i+1}^{(j)}\right)\right|=N k(n+m-i)-2 \mid N j+1$

For $j=2,4, \ldots, n-1$ and $j=\frac{k+1}{2}, \frac{k+3}{2}, \ldots, k$

$\left|\phi\left(u_{i}^{(j)}\right)-\phi\left(u_{i+1}^{(j)}\right)\right|=N k(n+m-i+1)-2 N j+1$

For $j=3,5, \ldots, n-2$ and $j=1,2, \ldots \frac{k-1}{2}$

$\left|\phi\left(u_{i+1}^{(j)}\right)-\phi\left(u_{i}^{(j)}\right)\right|=N k(n+m-i)-2 N j+1$

For $j=3,5, \ldots, n-2$ and $j=\frac{k+1}{2}, \frac{k+3}{2}, \ldots, k$

$\left|\phi\left(u_{i+1}^{(j)}\right)-\phi\left(u_{i}^{(j)}\right)\right|=N k(n+m-i+1)-2 N j+1$

This shows that the edges have the distinct labels $\{1, N+1,2 N+1, \ldots, N(q-1)+1\}$. 
International journal on applications of graph theory in wireless ad hoc networks and sensor networks (GRAPH-HOC) Vol.6, No.2, June 2014

$\mathrm{It}$, is clear from the ahove labelling that the funcion $\phi$ from the vertex set of $G$ los $\{0,1, N,(N+$ 1), $2 N,(2 N+1), \ldots, N(q-1), N(q-1)+1\}$ is in such a way that (i) $\phi$ is $1-1$ (ii) $\phi$ induces a bijection $\phi^{*}$ from the edge set of $G$ to $\{1, N+1,2 N+1, \ldots, N(q-1)+1\}$ where $\phi^{*}(u v)=|\phi(u)-\phi(v)|$. Hence the regular bamboo tree is one modulo $N$ graceful.

Clearly $\phi$ defines a one modulo $N$ graceful labelling of regular bamboo tree.

Example 2.5. One modulo 5 graceful labelling of regular bamboo tree. $(\mathrm{k}=5, \mathrm{n}=5, \mathrm{~m}=3)$

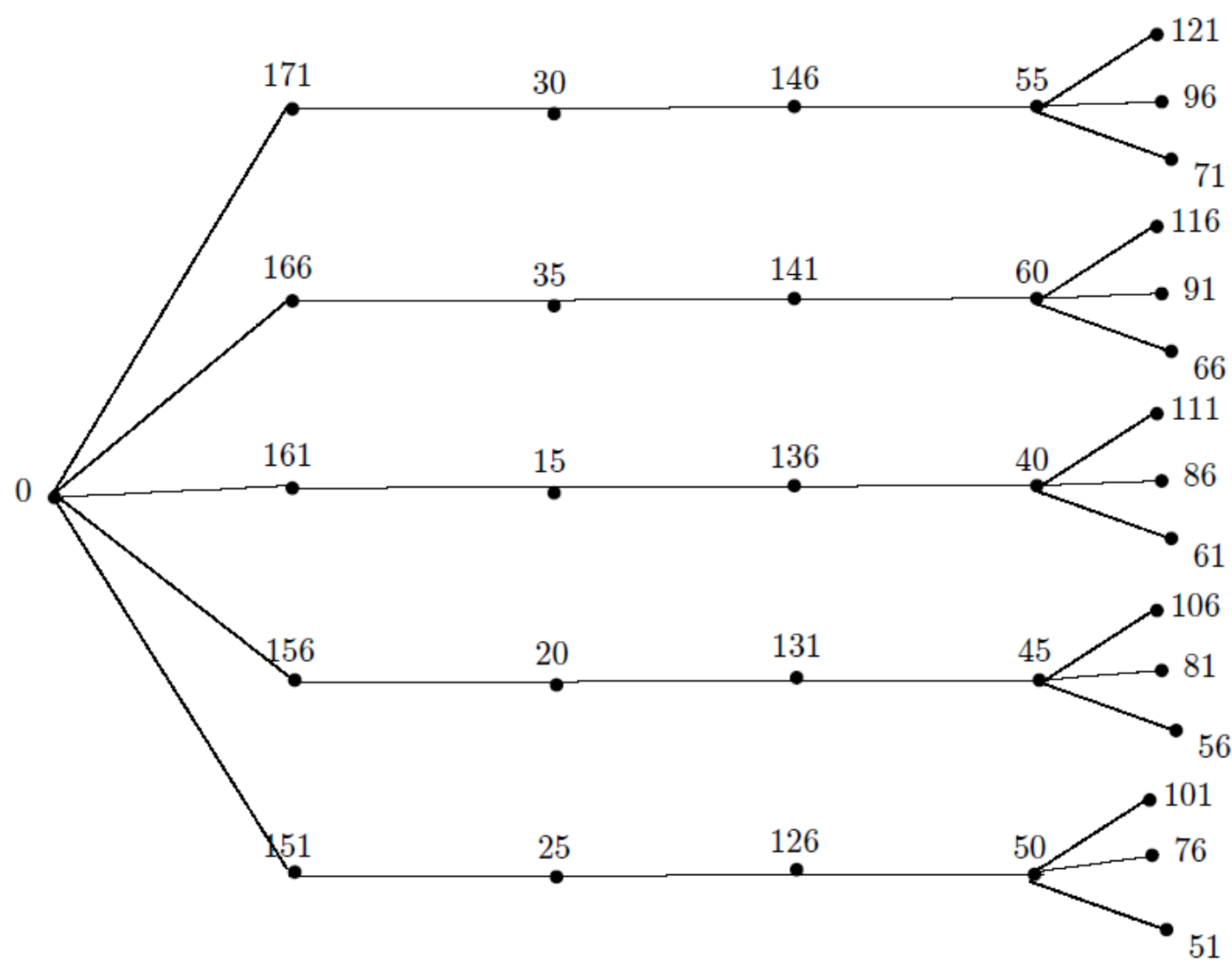

Case (ii) $\mathrm{k}$ is odd and $\mathrm{n}$ is even

Define 
International journal on applications of graph theory in wireless ad hoc networks and sensor networks

$\phi\left(u_{0}\right)=\phi\left(u_{1}^{(\jmath)}\right)=0$

(GRAPH-HOC) Vol.6, No.2, June 2014

For $i=2,4, \ldots, n$

$\phi\left(u_{i}^{(j)}\right)=N k(n+m-1)-(N-1)-N(j-1)-\frac{N k(i-2)}{2}$ for $j=1,2, \ldots, k$

For $i=3,5, \ldots, n-1$

$\phi\left(u_{i}^{(j)}\right)=\left\{\begin{array}{l}N(k+1)+N(j-1)+\frac{N k(i-3)}{2} \\ \frac{N(k+1)}{2}+N\left(j-\frac{(k+1)}{2}\right)+\frac{N k(i-3)}{2}\end{array}\right.$

For $r=1,2, \ldots, m$

$\phi\left(v_{r}^{(j)}\right)= \begin{cases}\frac{N(k n+2)}{2}+N k(r-1)+N(j-1) & \text { for } j=1,2, \ldots, \frac{(k-1)}{2} \\ \frac{N(k(n-1)+1)}{2}+N k(r-1)+N\left(j-\frac{(k+2)}{2}\right) & \text { for } j=\frac{(k+1)}{2}, \frac{(k+3)}{2}, \ldots, k\end{cases}$

for $j=1,2, \ldots, \frac{(k-1)}{2}$

for $j=\frac{(k+1)}{2}, \frac{(k+3)}{2}, \ldots, k$

The proof is similar to the proof in case(i).

Clearly $\phi$ defines a one modulo $N$ graceful labelling of regular bamboo tree.

Example 2.6. One modulo 8 graceful labelling of regular bamboo tree. $(k=3, n=6, m=2)$

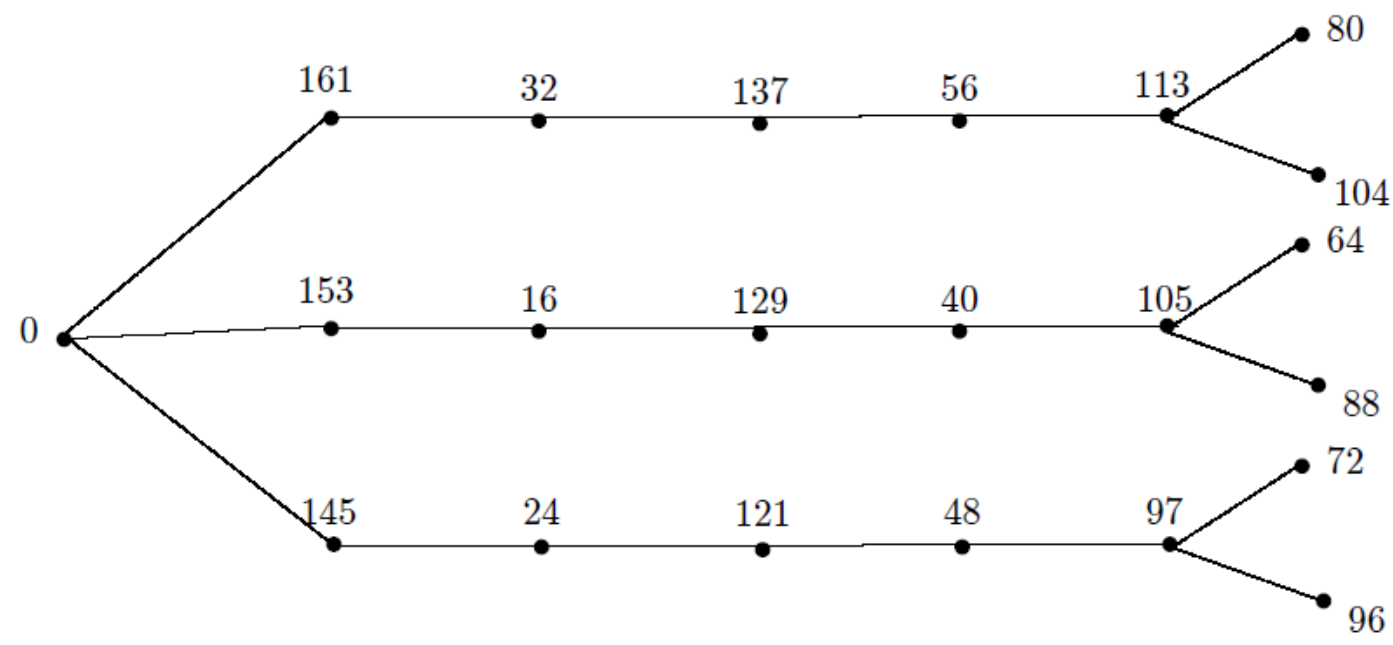

Case (iii) $\mathrm{k}$ is even and $\mathrm{n}$ is odd

Define 
International journal on applications of graph theory in wireless ad hoc networks and sensor networks (GRAPH-HOC) Vol.6, No.2, June 2014

$\phi\left(v_{r}^{(1)}\right)=N(r-1) \quad$ for $r=1,2, \ldots, m$

$\phi\left(u_{0}\right)-N k(n+m-1)-(N-1)-\frac{N(n-1)}{2}$

$\phi\left(\boldsymbol{u}_{i}^{(1)}\right)- \begin{cases}N(m-1)+\frac{N(n-1)}{2}-\frac{N(i-2)}{2} & \text { for } i=2,4, \ldots, n-1 \\ N k(m+n-1)+1-\frac{N(n-1)}{2}+\frac{N(i-3)}{2} & \text { for } i-3,5, \ldots, n\end{cases}$

For $i-3,5, \ldots, n$

$\phi\left(\boldsymbol{u}_{i}^{(j)}\right)=N k(n \mid m, 1) \quad\left(\begin{array}{llllll}N & 1\end{array}\right) \quad N\left(\begin{array}{lll}j & 2\end{array}\right) \quad \begin{gathered}N(n-1+k) \\ 2\end{gathered} \quad \begin{gathered}N(k-1)(i-3) \\ 2\end{gathered}$ for $j=2,3, \ldots, k$

For $i=2,4, \ldots, n-1$

$\phi\left(u_{i}^{(j)}\right)- \begin{cases}N m+\frac{N(n-1+k)}{2}+N(j-2)+\frac{N(k-1)(z-2)}{2} & \text { lor } j-2,3, \ldots, \frac{k}{2} \\ N(m-1)+\frac{N(n-1)}{2}+N+N\left(j-\frac{k}{2}-1\right)+\frac{N(k-1)(i-2)}{2} & \text { for } j-\frac{k}{2}+1, \frac{k}{2}+2, \ldots, k\end{cases}$

For $r=1,2, \ldots, m$

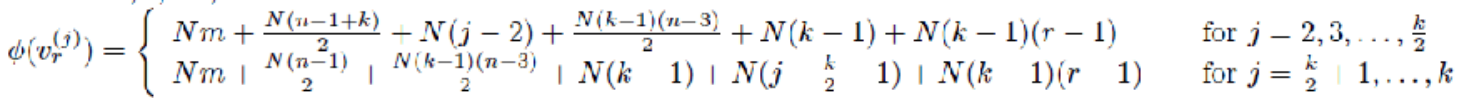

The proof is similar to the proof in case(i).

Clearly $\varphi$ defines a one modulo $\mathrm{N}$ graceful labelling of regular bamboo tree.

Example 2.7. One modulo 5 graceful labelling of regular bamboo tree. $(\mathrm{k}=6, \mathrm{n}=7, \mathrm{~m}=3)$

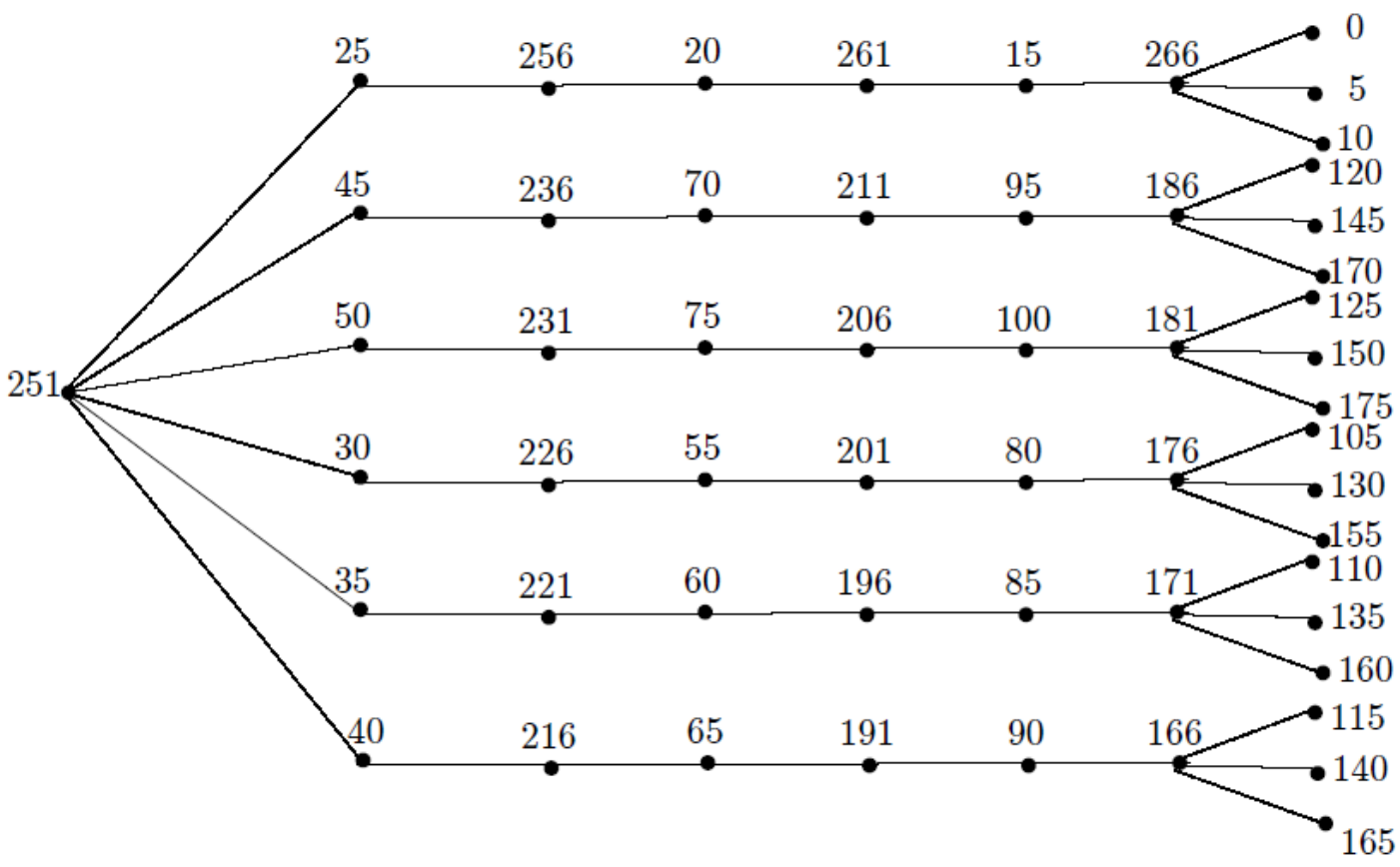

Case (iv) $\mathrm{k}$ is even and $\mathrm{n}$ is even 
International journal on applications of graph theory in wireless ad hoc networks and sensor networks (GRAPH-HOC) Vol.6, No.2, June 2014

Define

$\phi\left(v_{r}^{(1)}\right)=N(r \quad 1)$ for $r=1,2, \ldots, m$

$\phi\left(u_{0}\right)-N^{\prime}(m-1)-\frac{N r_{n}}{2}$

$\phi\left(u_{i}^{(1)}\right)= \begin{cases}N k(m+n-1)-(N-1)-\frac{N(n-2)}{2}+\frac{N(i-2)}{2} & \text { for } i=2,4, \ldots, n \\ N(m-1)+\frac{N(u-2)}{2}-\frac{N(i-3)}{2} & \text { for } i=3,5, \ldots, n-1\end{cases}$

For $i=2,4, \ldots, n$

$\phi\left(u_{i}^{(j)}\right)=N k\left(\begin{array}{llll}n \mid m & 1\end{array}\right) \quad\left(\begin{array}{lll}2 N & 1\end{array}\right) \quad N(j \quad 2) \quad \underset{2}{N(u-2)} \quad \underset{2}{N(k-1)(i-2)}$ for $j=2,3, \ldots, k$

For $i-3,5, \ldots, n-1$

$\phi\left(u_{i}^{(j)}\right)= \begin{cases}N m+N(n+k) \\ N m(j-2)+N\left(\begin{array}{l}k \\ 2\end{array}-1\right)+N(k-1)(i-3) & \text { for } j=2,3, \ldots, k \\ N m+\frac{N(n \mid K)}{2}+N\left(j-\frac{k}{2}-1\right)+\frac{N(k 1)(i 3)}{2} & \text { for } j-\frac{k}{2}+1, \frac{k}{2}+2, \ldots, k\end{cases}$

For $r-1,2, \ldots, m$

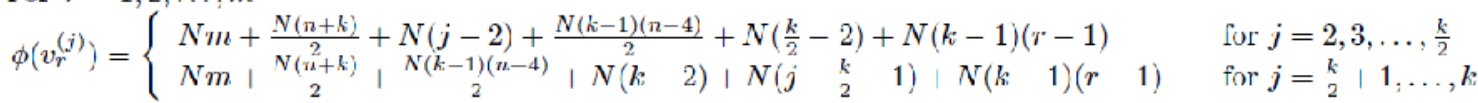

The proof is similar to the proof in case(i).

Clearly $\varphi$ defines a one modulo $\mathrm{N}$ graceful labelling of regular bamboo tree.

Example 2.8. One modulo 10 graceful labelling of regular bamboo tree. $(\mathrm{k}=8, \mathrm{n}=6, \mathrm{~m}=2)$

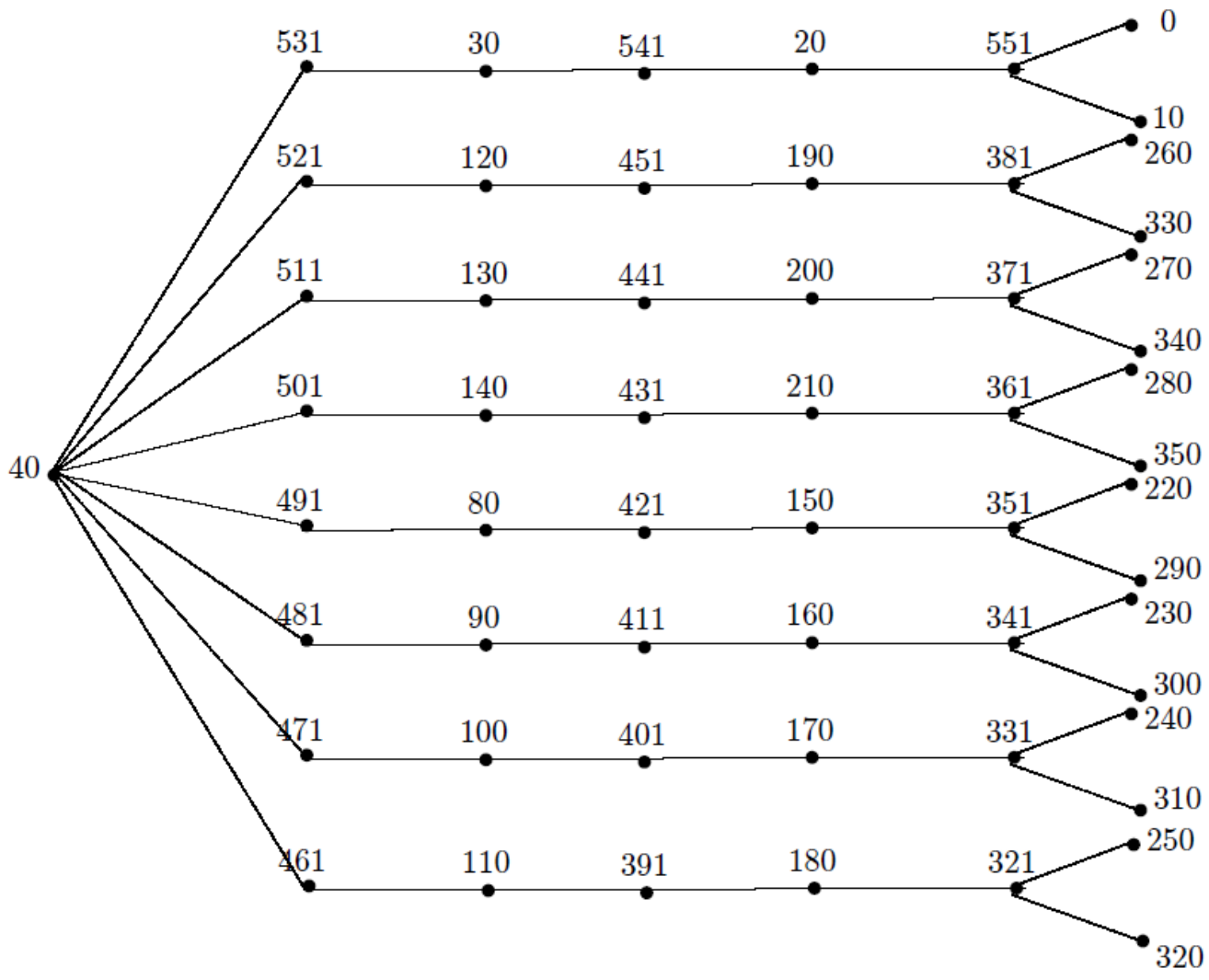


International journal on applications of graph theory in wireless ad hoc networks and sensor networks (GRAPH-HOC) Vol.6, No.2, June 2014

Theorem 2.9. Coconut tree is one modulo $N$ graceful for every positive integer $N$.

Proof: Let $u_{1}, u_{2}, \ldots, u_{\imath}$ be the vertices of a path $P_{r \iota}$ and $v_{1}, v_{2}, \ldots, v_{m}$ be the pendent vertices being adjaccnt with $u_{1}$ in the coconut trec $G$. Let $c_{i}$ denote the edge $u_{i} u_{i+1}$ of $P_{n}$ for $1<i<n \quad 1$ and $u_{1} v_{i}$ for $1 \leq i \leq m$. The coconut tree $G$ has $m+n$ vertices and $m+n-1$ edges.

Case (i) $n$ is odd.

Let $n=2 k \mid 1, k>1$.

Define

$\phi\left(u_{2 i-1}\right)=N(i-1) \quad$ for $i=1,2,3, \ldots, k+1$

$\phi\left(u_{2 i}\right)-2 N k-(N-1)-N(i-1)$ for $i-1,2,3, \ldots, k$

$\phi\left(v_{i}\right)=2 N k+1+N(i-1)$ for $i=1,2,3, \ldots, m$

From the definition of $\phi$ it is clear that

$\left\{\phi\left(u_{i}\right), i=1,2, \ldots, n\right\} \cup\left\{\phi\left(v_{i}\right), i=1,2, \ldots, m\right\}=\{0, N, 2 N, \ldots, N k\} \cup\{N(2 k-1)+1, N(2 k-$ $2)+1, \ldots, N k+1\} \cup\{N(2 k)+1, N(2 k+1)+1, \ldots, N(2 k+m-1)+1\}$

Thus it is clear that the vertices have distinct labels. Therefore $\varphi$ is $1-1$.

We compute the edge labelling in the following sequence.

For $1 \leq i \leq m$

$\left|\phi\left(v_{i}\right)-\phi\left(u_{1}\right)\right|-N(2 k+i-1)+1$

For $1 \leq i \leq k$

$\left|\phi\left(u_{2 i}\right)-\phi\left(u_{2 i-1}\right)\right|=N(2 k+1-2 i)+1$

$\left|\phi\left(u_{2 i}\right)-\phi\left(u_{2 i \mid 1}\right)\right|=N(2 k-2 i)+1$

This shows that the edges have the distinct labels $\{1, N+1,2 N+1, \ldots, N(q-1)+1\}$.

It is clcar from the above labclling that the function $\phi$ from the vertex sct of $G$ to $\{0,1, N,(N)$ 1) $, 2 N,(2 N+1), \ldots, N(q-1), N(q-1)+1\}$ is in such a way that $(i) \phi$ is $1-1$ (ii) $\phi$ induces a bijection $\phi^{*}$ from the edge set of $G$ to $\{1, N+1,2 N+1, \ldots, N(q-1)+1\}$ where $\phi^{*}(u v)=|\phi(u)-\phi(v)|$. Hence the coconut tree is one modulo $N$ graceful.

Clearly $\phi$ defines a one modulo $N$ graceful labelling of coconut tree.

Example 2.10. One modulo 3 graceful labelling and graceful labelling of coconut tree 
International journal on applications of graph theory in wireless ad hoc networks and sensor networks (GRAPH-HOC) Vol.6, No.2, June 2014
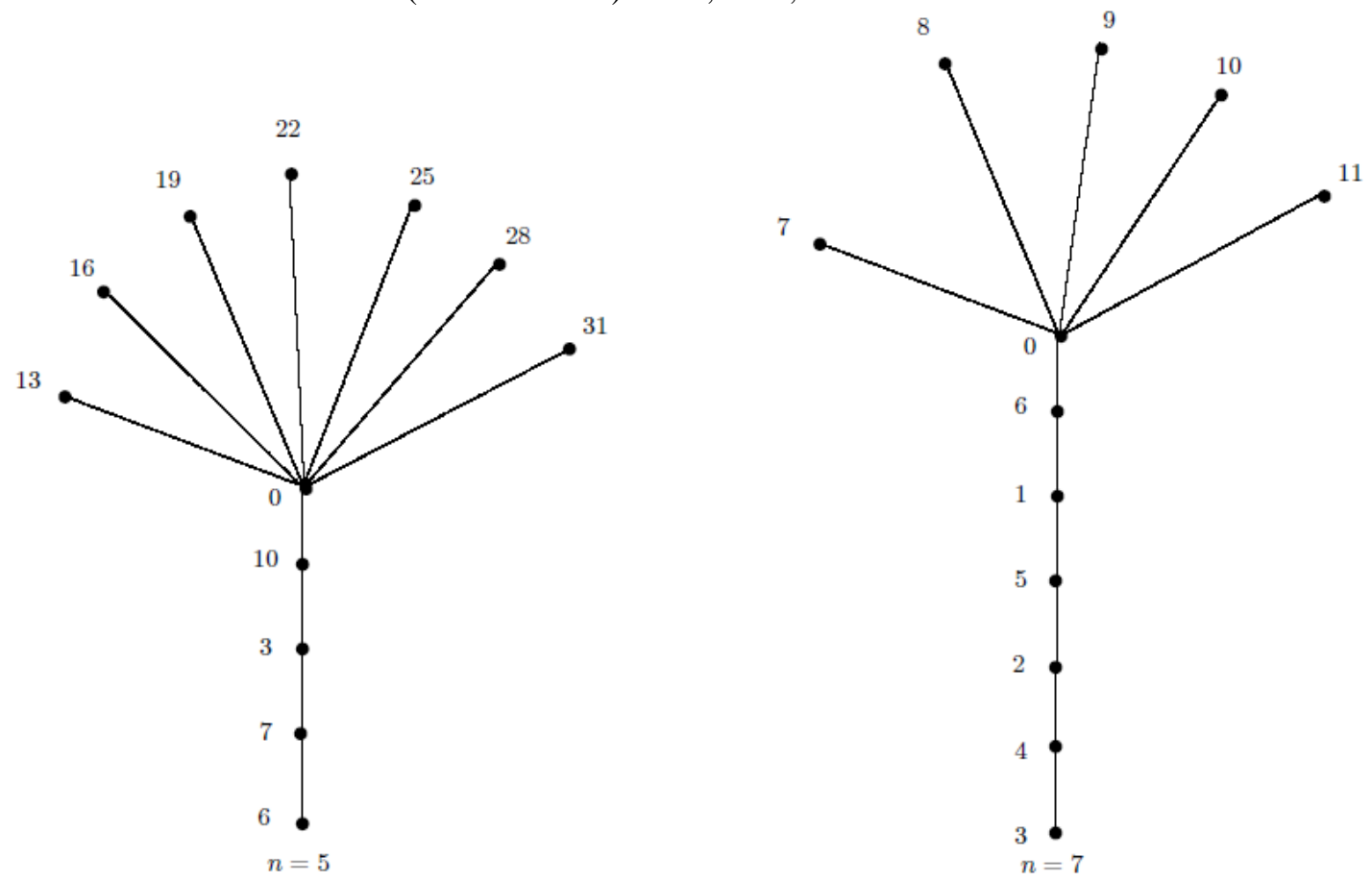

Case (ii) $n$ is even.

Let $n=2 k$.

Define

$\phi\left(u_{2 i-1}\right)=N(i-1)$ for $i=1,2,3, \ldots, k$

$\phi\left(u_{2 i}\right)=2 N k-(2 N-1)-N(i-1)$ for $i=1,2,3, \ldots, k$

$\phi\left(v_{i}\right)=2 N k+1+N(i-1) \quad$ for $i=1,2,3, \ldots, m$

The proof is similar to the proof in case(i).

Clearly $\phi$ defines a one modulo $N$ graceful labelling of coconut tree.

Example 2.11. One modulo 10 graceful labelling and odd graceful labelling of coconut tree. 
International journal on applications of graph theory in wireless ad hoc networks and sensor networks (GRAPH-HOC) Vol.6, No.2, June 2014
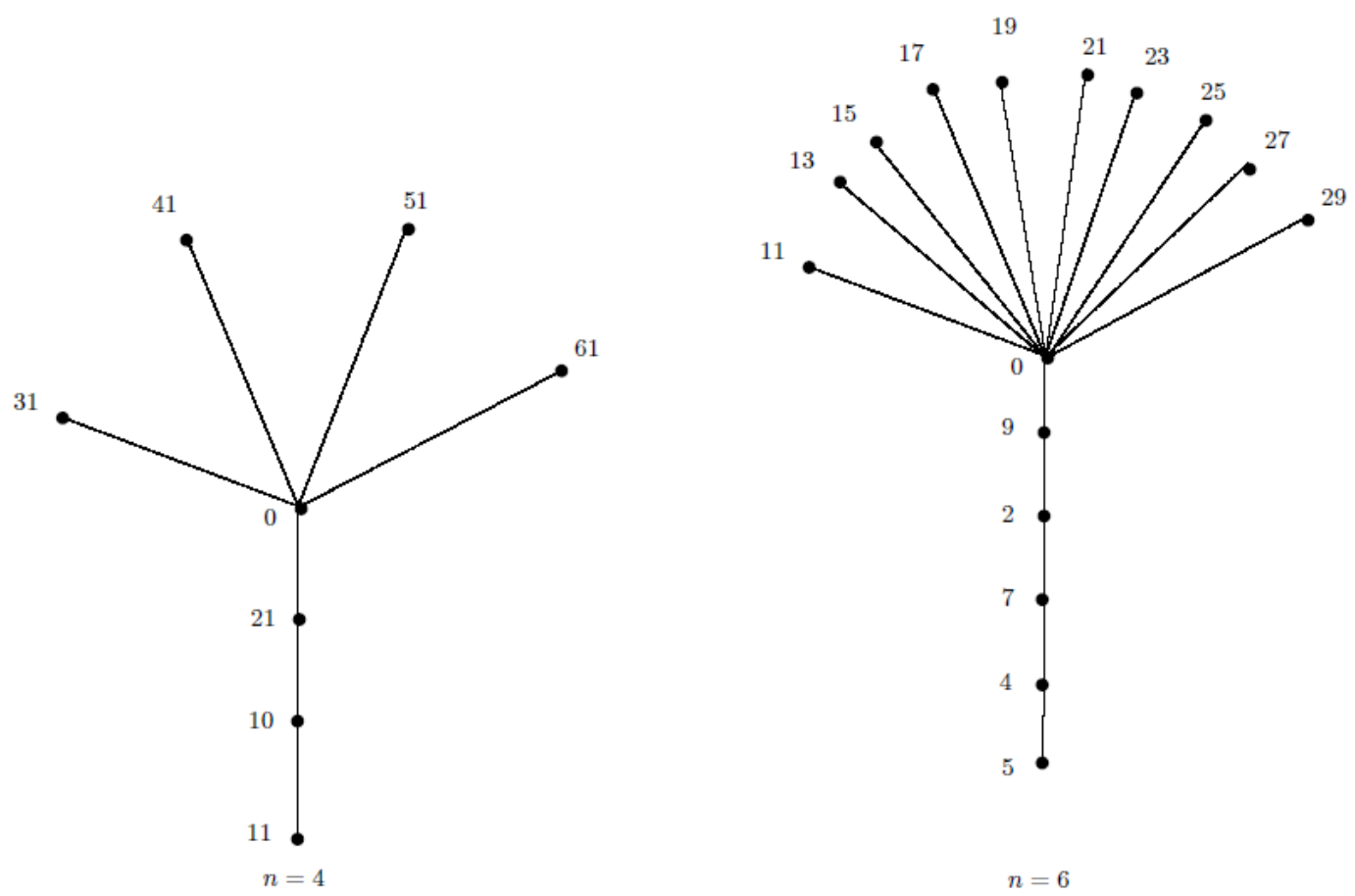

\section{References}

[1] R.B.Gnanajothi, Topics in Graph theory, Ph.D. Thesis, Madurai Kamaraj University, 1991.

[2] S.W.Golomb, How to number a graph in Graph theory and computing R.C. Read, ed., Academic press, New york (1972)23-27.

[3] Joseph A. Gallian, A Dynamic Survey of Graph Labeling, The Electronic Journal of Combinatorics, 18 (2011), \#DS6.

[4] V. Ramachandran and C. Sekar, One modulo N gracefulness of Acyclic graphs, Ultra Scientist of Physical Sciences, Vol.25 No (3)A, 417- 424 (2013).

[5] A.Rosa, On certain valuations of the vertices of a graph, Theory of graphs.(International Symposium, Rome July 1966)Gordom and Breach, N.Y and Dunod paris(1967)349-355.

[6] C.Sekar, Studies in Graph theory, Ph.D. Thesis, Madurai Kamaraj University, 2002.

[7] V.Swaminathan and C.Sekar, Gracefulness of Pa,b Ars Combinatoria,June 2004. 